\title{
XLV. Aggregates of minors of an axisymmetric determinant
}

\author{
Thomas Muir LL.D. C.M.G. F.R.S.
}

To cite this article: Thomas Muir LL.D. C.M.G. F.R.S. (1902) XLV. Aggregates of minors of an axisymmetric determinant , Philosophical Magazine Series 6, 3:16, 410-416, DOI: $10.1080 / 14786440209462781$

To link to this article: http://dx.doi.org/10.1080/14786440209462781

册 Published online: 15 Apr 2009.

Submit your article to this journal $[\pi$

Џ Article views: 3

Q View related articles $₫$ 
fluid any impurities which are detrimental to the film. In this way, I have been able to introduce ligroin in quantities sufficient to thoroughly wet the inside of the bulb, and have obtained in this way colours of even greater brilliancy than before.

\section{Conclusion.}

At the present stage of the work, it seems to me that it is impossible to decide either in favour of, or against, the theory of resonance. Most of the changes produced by varying the conditions seem to be what we should expect. The idea of electrical resonance has, at all events, proved useful as a working hypothesis, for it led directly to the discovery of the cause of the changes produced by low temperatures. While I have obtained coloured films from sodium, potassium, and lithium, I have been unable to obtain them from thalium and cadmium, which give granular deposits very similar in other respects. I cannot belp feeling that I shall eventually find some other explanation than the one ventured in the title of this paper, though I have been unable to conceive of any arrangement of media which will show colours of this nature by interference. At all events, it appears to me that we have colour phenomena quite unlike any that are already known, and which are of some interest, regardless of the ultimate explanation which may be given them.

Baltimore, February 1, 1.902.

XLV. Aggregates of Minors of an Axisymmetric Determinant. By Thomas Muik, LL.D., C.M.G., F.R.S.*

1. $\mathbf{H}^{\mathrm{OR}}$ the purpose of stating Kronecker's theorem regarding certain aggregates of minors of an axisymmetric determinant of even order, there does not seem to be a more appropriate notation than that introduced in the Philosophical Magazine $\dagger$ in 1884 , which consists in giving a typical term of the aggregate in question and adding some distinguishing mark to the letters or figures appearing therein whose variation gives rise to the other terms. Thus, the aggregate

$$
\left|\begin{array}{l}
1234 \\
5678
\end{array}\right|-\left|\begin{array}{l}
1235 \\
4678
\end{array}\right|+\left|\begin{array}{l}
1236 \\
4578
\end{array}\right|-\left|\begin{array}{l}
1237 \\
4568
\end{array}\right|+\left|\begin{array}{l}
1238 \\
4567
\end{array}\right|
$$

* Communicated by the Author.

+ The article is entitled "An Overlooked Discoverer in the Theory of Determinants," and appears in vol. xviii. pp. 416-427. 
Minors of an Axisymmetric Determinant.

which according to Kronecker vanishes when the parent determinant $\left|\begin{array}{l}12345678 \\ 12345678\end{array}\right|$ is axisymmetric, may be written

$$
\Sigma\left|\begin{array}{l}
123 \overline{4} \\
5678
\end{array}\right|
$$

it being understood that in each term under the sign of summation the row-numbers are taken in natural order and the column-numbers in order, and that the sign-factor of the term is $(-1)^{v}$ where $v$ is the number of inverted-pairs in the single line of numbers formed by writing the column-numbers immediately after the row-numbers.

2. Kronecker's theorem was originally published in 1882 , and since that date considerable additional light has been thrown on it, including such light as comes from generalization. The first generalization was pointed out in $1897 *$ and the second in $1901 \dagger$. The latter, due to Professor Metzler, is noteworthy because of its width; and on this and other grounds deserves some special attention such as the present paper aims at giving to it. In effect, Professor Metzler says that not only $\Sigma\left|\begin{array}{l}123 \overline{4} \\ 5678\end{array}\right|$ vanishes in the circumstances referred to, but also $\Sigma\left|\begin{array}{l}12 \overline{34} \\ 5678\end{array}\right|$ and $\Sigma\left|\begin{array}{l}\mathbf{1 2 3 4} \\ \mathbf{5 6 7 8}\end{array}\right|$; in other words, that Kronecker need not have confined the proposition to the case where $n-1$ of the row-numbers are invariable, because all that is necessary is that the number of such invariable row-numbers shall be greater than 0 and less than $n$. The mode of investigation is that employed in my second proof of Kronecker's theorem, viz., expansion of each member of the aggregate in terms of products of complementary minors and re-condensation resulting from the union of such of these products as have a factor in common.

3 . The main point calling for notice in the new generalization is the manner in which the vanishing of the aggregates comes about. A slight examination of a special case makes clear that in this respect there is an essential difference between the new and the old, and that further investigation is

* Transactions Roy. Soc. Edinburgh, vol. xxxix. p. 226 .

+ Transactions American Math. Soc. vol. ii. pp. 395-403.

2 E 2 

therefore called for. Let us consider the case of $\Sigma\left|\begin{array}{l}1 \overline{234} \\ \text { In less compact notation it is }\end{array}\right|$.

$$
\begin{aligned}
&\left|\begin{array}{l}
1234 \\
5678
\end{array}\right|-\left|\begin{array}{l}
1235 \\
4678
\end{array}\right|+\left|\begin{array}{l}
1236 \\
4578
\end{array}\right|-\left|\begin{array}{l}
1237 \\
4568
\end{array}\right|+\left|\begin{array}{l}
1238 \\
4567
\end{array}\right| \\
&+\left|\begin{array}{l}
1245 \\
3678
\end{array}\right|-\left|\begin{array}{l}
1246 \\
3578
\end{array}\right|+\left|\begin{array}{l}
1247 \\
3568
\end{array}\right|-\left|\begin{array}{l}
1248 \\
3567
\end{array}\right|+\left|\begin{array}{l}
1256 \\
3478
\end{array}\right| \\
&-\left|\begin{array}{l}
1257 \\
3468
\end{array}\right|+\left|\begin{array}{l}
1258 \\
3467
\end{array}\right|+\left|\begin{array}{l}
1267 \\
3458
\end{array}\right|-\left|\begin{array}{l}
1268 \\
3457
\end{array}\right|+\left|\begin{array}{l}
1278 \\
3456
\end{array}\right| \\
&-\left|\begin{array}{l}
1345 \\
2678
\end{array}\right|+\left|\begin{array}{l}
1346 \\
2578
\end{array}\right|-\left|\begin{array}{l}
1347 \\
2568
\end{array}\right|+\left|\begin{array}{l}
1348 \\
2567
\end{array}\right|-\left|\begin{array}{l}
1356 \\
2478
\end{array}\right| \\
&+\left|\begin{array}{l}
1357 \\
2468
\end{array}\right|-\left|\begin{array}{l}
1358 \\
2467
\end{array}\right|-\left|\begin{array}{l}
1367 \\
2458
\end{array}\right|+\left|\begin{array}{l}
1368 \\
2457
\end{array}\right|-\left|\begin{array}{l}
1378 \\
2456
\end{array}\right| \\
&+\left|\begin{array}{l}
1456 \\
2378
\end{array}\right|-\left|\begin{array}{l}
1457 \\
2368
\end{array}\right|+\left|\begin{array}{l}
1458 \\
2367
\end{array}\right|+\left|\begin{array}{l}
1467 \\
2358
\end{array}\right|-\left|\begin{array}{l}
1468 \\
2357
\end{array}\right| \\
&+\left|\begin{array}{l}
1478 \\
2356
\end{array}\right|-\left|\begin{array}{l}
1567 \\
2348
\end{array}\right|+\left|\begin{array}{l}
1568 \\
2347
\end{array}\right|-\left|\begin{array}{l}
1578 \\
2346
\end{array}\right|+\left|\begin{array}{l}
1678 \\
2345
\end{array}\right|,
\end{aligned}
$$

the number of terms being of course $\mathrm{C}_{7.3}, i . e .35$. This may be viewed as consisting of four sub-aggregates, A, B, C, D, viz. A occupying the first line, $B$ the second and third lines, $\mathrm{C}$ the fourth and fifth lines, and $\mathrm{D}$ the sixth and seventh lines. Of these the first is seen to be a Kronecker aggregate. The second B consists of two parts which cancel each other; for the sum of the first four terms being equal to $\left|\begin{array}{l}1243 \\ 5678\end{array}\right|$ diminished by a Kronecker aggregate is equal to $-\left|\begin{array}{l}1234 \\ 5678\end{array}\right|$, and the sum of the last six terms can be shown to be $\left|\begin{array}{l}1234 \\ 5678\end{array}\right|$. Similarly, the sum of the first four terms of C cancels the sum of the last six terms: and the sum of the first six terms of $D$ cancels the sum of the last four terms. The new aggregate, $\Sigma\left|\begin{array}{l}\mathbf{1 2 3 4} \\ \mathbf{5 6 7 8}\end{array}\right|$, is thus seen to be the sum of four zeros; and it will be found that all the others have a like constitution.

4. The four sub-aggregates in the preceding are easily seen to be

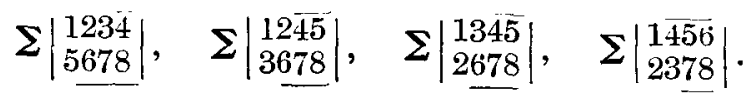

The last three are not essentially different; so that to 
Kronecker's fundamental aggregate $\Sigma\left|\begin{array}{l}123 \overline{4} \\ 5678\end{array}\right|$ there has to be added one other, $\Sigma\left|\begin{array}{l}12 \overline{45} \\ 3678\end{array}\right|$, the common characteristic of the two being the invariability of the line-numbers $1,2,3$.

The vanishing of this new aggregate, $\mathbf{\Sigma}\left|\begin{array}{l}12 \overline{4} \overline{5} \\ 3678\end{array}\right|$, is established by separating the four terms of it which have 4 as a row-number from the six terms which have 4 as a column-number, and showing that the one group cancels the other. Thus

$$
\begin{aligned}
\Sigma\left|\begin{array}{l}
12 \overline{45} \\
3678
\end{array}\right| & =\Sigma\left|\begin{array}{l}
124 \overline{5} \\
3678
\end{array}\right|+\Sigma\left|\begin{array}{l}
12 \overline{56} \mid \\
3478
\end{array}\right|, \\
& =\left|\begin{array}{c}
1243 \\
5678
\end{array}\right|+\left|\begin{array}{l}
1234 \\
5678
\end{array}\right|, \\
& =0 .
\end{aligned}
$$

The penultimate step here presents two expressions for $5678 \mid$. The second of these is new; the first is not, since the vanishing of Kronecker's aggregate $\Sigma\left|\begin{array}{l}123 \overline{4} \\ 5678\end{array}\right|$ may also be viewed as a statement that

$$
\left|\begin{array}{l}
1234 \\
5678
\end{array}\right|=\Sigma\left|\begin{array}{l}
123 \overline{5} \\
4678
\end{array}\right|
$$

where, as is often convenient in dealing with sub-aggregates, the typical term on the right is reckoned positive and the others determined from it. We are thus led to a series of identities

$$
\left|\begin{array}{l}
1234 \\
5678
\end{array}\right|=\Sigma\left|\begin{array}{l}
123 \overline{5} \\
4678
\end{array}\right|=\Sigma\left|\begin{array}{l}
12 \overline{56} \\
3478
\end{array}\right|=\Sigma\left|\begin{array}{l}
1 \overline{567} \\
2348
\end{array}\right| .
$$

5. If in the case of five-line minors we denote the fundamental Kronecker aggregate,

$$
\Sigma\left|\begin{array}{l}
1234 \overrightarrow{5} \\
6789 \tau
\end{array}\right| \text { by } \mathrm{K}
$$

the Metzler aggregates

$$
\Sigma\left|\begin{array}{l}
123 \overline{45} \\
6789 \tau
\end{array}\right|, \quad \Sigma\left|\begin{array}{l}
123 \overline{45} \\
6789 \tau
\end{array}\right|, \quad \Sigma\left|\begin{array}{l}
\overline{1.345} \\
6789 \tau
\end{array}\right|, \text { by } \mathrm{M}_{1}, \mathrm{M}_{2}, \mathrm{M}_{3} ;
$$


and the still more recent aggregates

by

$$
\Sigma\left|\begin{array}{l}
123 \overline{56} \\
4789 \tau
\end{array}\right|, \quad \Sigma\left|\begin{array}{l}
12 \overline{567} \\
3489 \tau
\end{array}\right|, \quad \Sigma\left|\begin{array}{l}
12 \overline{456} \\
3789 \tau
\end{array}\right|, \quad \Sigma\left|\begin{array}{l}
1 \overline{3456} \\
2789 \tau
\end{array}\right|
$$

$\mu_{1}, \quad \mu_{2}, \quad \mu_{3}, \quad \mu_{4} ;$

the following partition of the 126 terms of $\Sigma\left|\begin{array}{l}1 \overline{2345} \\ 6789 \tau\end{array}\right|$ will make clear the various relationships involved.

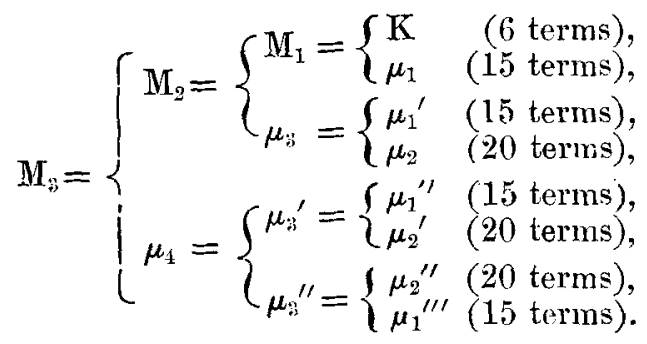

Of course for perfect completeness we should have started with the 252 terms of the aggregate $\Sigma|\underline{6789 \tau}|$, dividing it into $\Sigma\left|\begin{array}{l}1 \overline{2345} \\ 6789 \tau\end{array}\right|$ and $\Sigma\left|\begin{array}{l}2345 \overline{6} \\ 1789 \tau\end{array}\right|$, - that is to say, into $M_{3}$ and $\mathrm{M}_{3}^{\prime}$ : but nothing would thereby have been gained, as the difference between the one half and the other is made to disappear by row-and-column interchange.

The fundamental aggregates in the collection are seen to be $\mathrm{K}, \mu_{1}, \mu_{2}$; that is to say,

$$
\Sigma\left|\begin{array}{l}
1234 \overline{5} \\
6789 \tau
\end{array}\right|, \quad \Sigma\left|\begin{array}{l}
123 \overline{56} \\
4789 \tau
\end{array}\right|, \quad \Sigma\left|\begin{array}{l}
12 \overline{567} \\
3489_{\tau}
\end{array}\right|,
$$

the others on the extreme right being variants of two of these and therefore indicated by the same letters with one or more dashes. The common characteristic of the three is the invariability of four line-numbers, all the possible distributions of four such numbers among rows and columns being

$$
4+0, \quad 3+1, \quad 2+2 .
$$

6. The proof that $\mu_{1}$ and $\mu_{2}$ vanish on the imposition of axisymmetry is accomplished by continuing in each case the process of fission by which they themselves arose, and then 
showing that the two components differ only in sign. Thus the fifteen-termed aggregate

$$
\begin{aligned}
& \mu_{1}^{\prime}, \text { i. e. } \Sigma\left|\begin{array}{l}
1245 \overline{6} \\
3789_{\tau}
\end{array}\right|, \\
&= \Sigma\left|\begin{array}{l}
1245 \overrightarrow{6} \\
3789_{\tau}
\end{array}\right|+\Sigma\left|\begin{array}{l}
124 \overrightarrow{67} \\
3589_{\tau}
\end{array}\right|, \\
&=\left|\begin{array}{l}
12453 \\
6789_{\tau}
\end{array}\right|+\left|\begin{array}{l}
12435 \\
6789_{\tau}
\end{array}\right|, \\
&=0:
\end{aligned}
$$

and the twenty-termed aggregate

$$
\begin{aligned}
\mu_{2}^{\prime \prime}, \text { i. e. } & \Sigma\left|\begin{array}{l}
14 \overline{567} \\
2389 \tau
\end{array}\right|, \\
= & \Sigma\left|\begin{array}{l}
145 \overline{67} \\
2389 \tau
\end{array}\right|-\Sigma\left|\begin{array}{l}
14 \overline{678} \\
2359 \tau
\end{array}\right|, \\
= & \left|\begin{array}{l}
14523 \\
6789 \tau
\end{array}\right|-\left|\begin{array}{l}
14235 \\
6789 \tau
\end{array}\right|, \\
= & 0 .
\end{aligned}
$$

The 35-termed aggregate $\mu_{3}$ and the 70-termed aggregate $\mu_{t}$ are seen to resemble the M's in their constitution, the one being the sum of two zeros and the other the sum of four.

7. Finally, let it be noted that two lines of generalization are berein marked out. The first is indicated in the series of identities-

$$
\begin{aligned}
& \left|\begin{array}{ll}
\alpha & \beta \\
\gamma & \delta
\end{array}\right|=\Sigma\left|\begin{array}{ll}
\alpha & \bar{\gamma} \\
\beta & \delta
\end{array}\right| \\
& \left|\begin{array}{lll}
\alpha & \beta & \gamma \\
\delta & \varepsilon & \zeta
\end{array}\right|=\Sigma\left|\begin{array}{lll}
\alpha & \beta & \bar{\delta} \\
\gamma & \epsilon & \zeta
\end{array}\right|=-\Sigma\left|\begin{array}{ll}
\alpha & \bar{\delta} \epsilon \\
\beta & \gamma \zeta
\end{array}\right|
\end{aligned}
$$

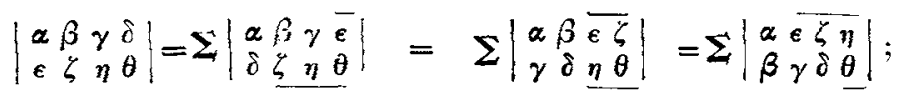

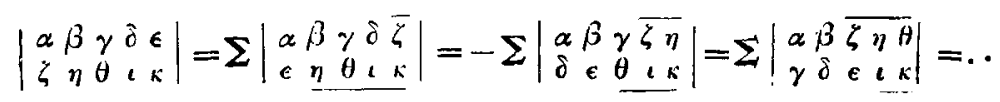

where the opening identity in each line is Kronecker's. The 
second is seen in the series,

$$
\begin{aligned}
& 0=\Sigma\left|\begin{array}{ll}
\alpha & \bar{\beta} \\
\gamma & \delta
\end{array}\right| \\
& 0=\Sigma\left|\begin{array}{lll}
\alpha & \beta & \bar{\gamma} \\
\delta & \epsilon & \zeta
\end{array}\right|=\Sigma\left|\begin{array}{lll}
\alpha & \bar{\gamma} \\
\beta & \underline{\delta} & \zeta
\end{array}\right|,
\end{aligned}
$$

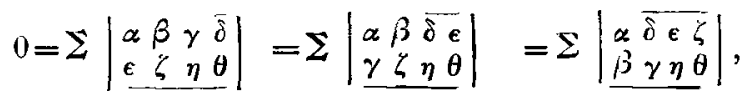

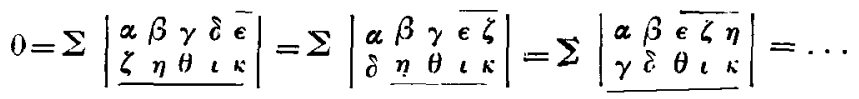

where again on the extreme left the same Kronecker identities appear.

Capetown, South Africa, 3rd February, 1902.

XLVI. On the Question of Hydrogen in the Atmosphere. $B y$ Lord RAYLEIGH, F.R.S.*

T T will be remembered that M. Armand Gautier, as the 1 result of very elaborate investigations, was led to the conclusion that air, even from the Atlantic, contains by volume nearly 2 parts in 10,000 of free hydrogen. The presence of so much hydrogen, nearly two thirds of the carbonic acid which plays such an important part, is of interest in connexion with theories pointing to the escape of light constituents from the planetary atmospheres. Besides the free hydrogen, M. Gautier found in the air of woors and towns considerable quantities of hydrocarbons yielding $\mathrm{CO}_{2}$ when led over hot copper oxide.

\section{Spectroscopic Evidence.}

In the Philosophical Magazine for Jan. 1901, I described some observations upon the spectrum of sparks taken in dried air at atmospheric pressure, which seemed "to leave a minimum of room for the hydrogen found by M. Gautier." Subsequently (April 1901), these experiments were repeated with confirmatory results. The spectra, taken from platinum points, of pure country air and the same to which $\frac{2}{10,000}$ of

* Communicated by the Author. 